San Jose State University

SJSU ScholarWorks

Faculty Publications

Physics and Astronomy

$1-1-2012$

\title{
The SLUGGS Survey: NGC 3115, a critical test case for metallicity bimodality in globular cluster systems
}

\author{
J. P. Brodie \\ University of California, Santa Cruz \\ C. Usher \\ Swinburne University \\ C. Conroy \\ University of California, Santa Cruz \\ J. Strader \\ Michigan State University \\ J. A. Arnold \\ University of California, Santa Cruz
}

See next page for additional authors

Follow this and additional works at: https://scholarworks.sjsu.edu/physics_astron_pub

Part of the Astrophysics and Astronomy Commons

\section{Recommended Citation}

J. P. Brodie, C. Usher, C. Conroy, J. Strader, J. A. Arnold, D. A. Forbes, and Aaron J. Romanowsky. "The SLUGGS Survey: NGC 3115, a critical test case for metallicity bimodality in globular cluster systems" Astrophysical Journal (2012). https://doi.org/10.1088/2041-8205/759/2/L33

This Article is brought to you for free and open access by the Physics and Astronomy at SJSU ScholarWorks. It has been accepted for inclusion in Faculty Publications by an authorized administrator of SJSU ScholarWorks. For more information, please contact scholarworks@sjsu.edu. 


\section{Authors}

J. P. Brodie, C. Usher, C. Conroy, J. Strader, J. A. Arnold, D. A. Forbes, and Aaron J. Romanowsky 


\title{
THE SLUGGS SURVEY: NGC 3115, A CRITICAL TEST CASE FOR METALLICITY BIMODALITY IN GLOBULAR CLUSTER SYSTEMS
}

\author{
Jean P. Brodie ${ }^{1}$, Christopher Usher ${ }^{2}$, Charlie Conroy ${ }^{1}$, Jay Strader $^{3}$, Jacob A. Arnold $^{1}$, \\ Duncan A. Forbes ${ }^{2}$, And Aaron J. RomanowsKy ${ }^{1,4}$ \\ ${ }^{1}$ University of California Observatories and Department of Astronomy and Astrophysics, University of California, Santa Cruz, CA 95064, USA; brodie@ ucolick.org \\ ${ }^{2}$ Centre for Astrophysics \& Supercomputing, Swinburne University, Hawthorn, VIC 3122, Australia \\ ${ }^{3}$ Department of Physics and Astronomy, Michigan State University, East Lansing, MI 48824, USA \\ ${ }^{4}$ Department of Physics and Astronomy, San José State University, One Washington Square, San Jose, CA 95192, USA \\ Received 2012 September 21; accepted 2012 September 26; published 2012 October 22
}

\begin{abstract}
Due to its proximity $(9 \mathrm{Mpc})$ and the strongly bimodal color distribution of its spectroscopically well-sampled globular cluster (GC) system, the early-type galaxy NGC 3115 provides one of the best available tests of whether the color bimodality widely observed in GC systems generally reflects a true metallicity bimodality. Color bimodality has alternatively been attributed to a strongly nonlinear color-metallicity relation reflecting the influence of hot horizontal-branch stars. Here, we couple Subaru Suprime-Cam gi photometry with Keck/DEIMOS spectroscopy to accurately measure GC colors and a CaT index that measures the Ca II triplet. We find the NGC 3115 GC system to be unambiguously bimodal in both color and the CaT index. Using simple stellar population models, we show that the $\mathrm{CaT}$ index is essentially unaffected by variations in horizontal-branch morphology over the range of metallicities relevant to GC systems (and is thus a robust indicator of metallicity) and confirm bimodality in the metallicity distribution. We assess the existing evidence for and against multiple metallicity subpopulations in early- and late-type galaxies and conclude that metallicity bi/multimodality is common. We briefly discuss how this fundamental characteristic links directly to the star formation and assembly histories of galaxies.
\end{abstract}

Key words: galaxies: individual (NGC 3115) - galaxies: star clusters: general - globular clusters: general

Online-only material: color figures

\section{INTRODUCTION}

The fact that most massive galaxies possess globular cluster (GC) systems that are bimodal in color has been recognized for over a decade (e.g., Zepf \& Ashman 1993; Ostrov et al. 1993). The general presence in galaxy GC systems of two principal subpopulations is implicit in this finding. It is now well established that the vast majority of GCs are old ( $\gtrsim 10 \mathrm{Gyr}$; e.g., Strader et al. 2005; Puzia et al. 2005), which suggests that the subpopulations are separated predominantly by metallicity rather than age.

Considerable effort has since been devoted to understanding the origin of metallicity bimodality as it has significant implications for understanding the star formation and assembly histories of galaxies (see review by Brodie \& Strader 2006). This follows from the notion that GC formation accompanies all major star formation events in a galaxy's history. Once formed, GCs remain as unchanging (compared to galaxy starlight) bright beacons, and are the accompanying witnesses to the development of cosmic structure.

This general view has been questioned, however. Richtler (2006) showed that a hypothetical unimodal metallicity distribution function (MDF) can appear bimodal in some optical colors when scatter is included in the color-metallicity relation. Yoon et al. (2006) suggested, based on their single stellar population (SSP) models, that a strong inflection appears in the central part of the optical color-metallicity relations (between $[\mathrm{Z} / \mathrm{H}]=-1.5$ and -0.5$)$, driven by contributions from horizontal-branch $(\mathrm{HB})$ stars. This inflection can transform a unimodal MDF into two color peaks in their models. Recently, Yoon et al. (2011a, 2011b) explored their expectations for ultraviolet/optical color combinations and concluded, again, that the underlying GC MDFs are generally unimodal. Recent investigations have found evidence for and against metallicity bimodality (Alves-Brito et al. 2011; Foster et al. 2010; Chies-Santos et al. 2012; Blakeslee et al. 2012) without a clear resolution of the issue.

The most obvious objection to the idea of a unimodal MDF for GCs is that it conflicts with our knowledge of the best-studied GC system of all, that of the Milky Way. Here, metallicities are tied to accurate abundance measurements for individual stars and the unambiguously bimodal metallicity distribution links directly to distinct spatial and kinematical GC subsystems (Searle \& Zinn 1978). Nonetheless, generalization to other galaxies must rely on more limited information, such as metallicities derived from integrated spectroscopy, as we cannot obtain full chemical and phase-space data for systems beyond the Local Group (although see Pota et al. 2012 for the results of a color-kinematical survey of nearby GC systems).

In principle, to resolve the bimodality question in a convincing way, we would need to identify galaxies having the most clearly bimodal color distributions and derive metallicities from spectra for a significant fraction of their GC subpopulations. Galaxies with unclear color bimodality tell us little about this particular issue, although they may be revealing about the complexities of galaxy assembly, as discussed below. If, in even one unequivocal case, a bimodal fit to the MDF were to be strongly preferred over a unimodal one, and the proportion of metal-rich to metal-poor GCs were to be similar to the red/blue number ratio, this would provide convincing support for the principle of distinct GC metallicity subpopulations in galaxies. Similarly, if even one case with strong color bimodality turned out to be unambiguously unimodal in metallicity, this would provide a huge boost to the unimodal metallicity hypothesis.

Here we present new spectroscopically based measures of GC metallicity in a nearby galaxy, NGC 3115 , that offers the best available test in an early-type galaxy of these distinct 
interpretations of color bimodality. NGC 3115, one of 11 nearby galaxies in the spectroscopic study of Usher et al. (2012), is an $\mathrm{S} 0$ galaxy hosting a populous GC system that is strongly bimodal in optical colors.

The great advantage of NGC 3115 for exploring the question of metallicity bimodality is its proximity $(\sim 9.4 \mathrm{Mpc}$; Tonry et al. 2001). This is important because spectroscopic studies that generate reliable metallicity estimates for GCs are very challenging, even for the largest telescopes equipped with efficient spectrographs. This has led to the tendency for spectroscopically derived metallicity estimates to be confined to the brighter subset of GCs in all but the closest galaxies. The brightest GCs are liable to display unimodal color and metallicity distributions because of the "blue tilt" phenomenon, or mass-metallicity relation, that reflects self-enrichment for massive metal-poor GCs (Strader et al. 2006; Harris et al. 2006; Mieske et al. 2006). The blue tilt tends to reduce the separation of the red and blue distributions with increasing brightness in the color-magnitude plane.

For NGC 3115, which has a populous GC system consisting of $546 \pm 80 \mathrm{GCs}$ within $50 \mathrm{kpc}$ (Faifer et al. 2011), we spectroscopically sample a significant fraction $(\sim 20 \%)$ of the ordinary GC population. Indeed, we obtain a reliably measured metallicity index, independent of both color and SSP modeling considerations, down to the turnover of the GC luminosity function.

Section 2 describes the new observations that allow us to characterize the metallicity distribution of NGC 3115 GCs. Section 3 presents colors and measurements of the metallicitysensitive CaII triplet index (CaT) for these GCs, and our investigation of the dependence of the CaT on HB morphology. Section 4 is a discussion of these findings in the context of arguments in the literature for and against metallicity bimodality. Section 5 contains the summary and conclusions.

\section{OBSERVATIONS}

To study the metallicity distribution of the NGC 3115 GC system, we use the Subaru Suprime-Cam gi photometry and the Keck/DEIMOS multiobject spectroscopy given by Arnold et al. (2011) but with the color corrections and revised zero points presented in Usher et al. (2012). These data were taken as part of the SLUGGS survey. ${ }^{5}$ The spectroscopic program was designed to target the region around the CaII triplet at 8498,8542 , and $8662 \AA$ (CaT). To negate the effect of the strong sky lines in this spectral region, we employ a technique described in detail in Foster et al. $(2010,2011)$ and fit a linear combination of stellar template spectra to the observed spectra while masking out those spectral regions containing significant sky lines. The fitted spectra are then continuum-normalized and the strength of the CaT index is measured from the normalized spectra. A Monte Carlo resampling technique is used to derive asymmetric confidence intervals for each measurement. Further details of the CaT observations and measurement techniques are given in Usher et al. (2012).

\section{GC METALLICITIES}

CaT has long been known to correlate with metallicity for old stellar populations (e.g., Armandroff \& Zinn 1988; Cenarro et al. 2002) but issues have been raised about its usefulness as a one-to-one predictor of metallicity because it may become less

\footnotetext{
5 http://sluggs.ucolick.org
}

sensitive ("saturate") at higher metallicities $([Z / H]>-0.4)$ and because it may respond to changes in HB morphology due to the presence of Paschen lines in the line index bandpasses (Foster et al. 2010). SSP modeling now shows that the flattening at high metallicities is mild, and that the effect of the HB is minimal (see below).

Figure 1 is a plot of the highest $\mathrm{S} / \mathrm{N}\left(>12 \AA^{-1}\right) \mathrm{CaT}$ measurements for 71 GCs in NGC 3115 as a function of $(g-i)_{0}$ color, along with the corresponding histograms. A cut of $\left(>8 \AA^{-1}\right)$ increases the sample to 122 but does not significantly change the robustness of the Gaussian Mixture Modeling (GMM) statistics (Muratov \& Gnedin 2010). This galaxy exhibits the clearest color bimodality of any known GC system ( $>99.9 \%$ confidence). Its DD value (a GMM measure of peak separation divided by peak widths) is 3.9. By comparison, bimodality "poster children" NGC 4594 and NGC 4472 have DD values of $3.2(B-R)$ and $3.0(g-z)$, respectively. Overplotted in the upper panel of Figure 1 is the color histogram for the entire NGC 3115 photometric sample, showing that the spectroscopically observed subset faithfully represents the overall GC distribution. The bimodal nature of both the color and the $\mathrm{CaT}$ index distributions is unambiguous.

Possible index sensitivity to the distribution of stars on the $\mathrm{HB}$ is investigated by measuring $\mathrm{CaT}$ in model spectra, using the same methods used for GC measurements. The models are based on those of Conroy and collaborators (Conroy et al. 2009; Conroy \& Gunn 2010), but have new synthetic spectral libraries to allow for $\alpha$-enhancement and explicit modeling of the CaT index. Two extreme cases are investigated in a 13 Gyr SSP, one with an extreme blue $\mathrm{HB}$ at all metallicities and the other with an all-red HB. Figure 2 demonstrates conclusively that the CaT index is effectively insensitive to $\mathrm{HB}$ morphology; while the spectra themselves change with the addition of hot stars, the effects are present in both the index bandpass and pseudocontinua and largely cancel out. Figure 2 also shows that $\alpha$-enhancement does not change the shape of the relation between metallicity and the CaT index, as the enhanced models simply move along the relation defined by the solar-scaled models. CaT is thus an accurate measure of total metallicity, $[Z / H]$, in GCs (where $[Z / H]=[\mathrm{Fe} / \mathrm{H}]+0.934 \times[\alpha / \mathrm{Fe}])$. The relation between $\mathrm{CaT}$ and $[Z / \mathrm{H}]$ is approximately linear and permits excellent metallicity discrimination over essentially the full range of observed GC metallicities (the models span $-2.0<[Z / \mathrm{H}]<0.0)$.

Figure 3 is a plot of $[Z / \mathrm{H}]$, derived from CaT measurements using the $\mathrm{CaT}-[\mathrm{Z} / \mathrm{H}]$ relation of Usher et al. (2012), against $(g-i)_{0}$ color. Usher et al. compared data and SSP models for a large number of galaxies' GC systems and defined a robust linear relation between $\mathrm{CaT}$ and $[\mathrm{Z} / \mathrm{H}]$. A GMM analysis finds the $[Z / \mathrm{H}]$ distribution of the NGC 3115 GCs to be bimodal at the $99.8 \%$ confidence level. Overplotted are the Yoon et al. (2011a) color-metallicity relation and a two-component linear fit to the NGC 3115 data. The broken linear fit provides a better representation of the data. A similar result was found for M31 GCs, where Peacock et al. (2011) used ugriz optical photometry and spectroscopic metallicities and found color-metallicity relations that are all approximately linear, without the sharp inflections predicted by the Yoon et al. (2011a) models.

Figure 4 presents a comparison of the MDF derived from the CaT index with MDFs obtained by applying the Yoon et al. (2011a) model, and the Usher et al. (2012) empirical color $-[\mathrm{Z} / \mathrm{H}]$ relation (their Equation (10)), to the observed $(g-i)_{0}$ colors. While the Yoon et al. color $-[Z / H]$ relation 

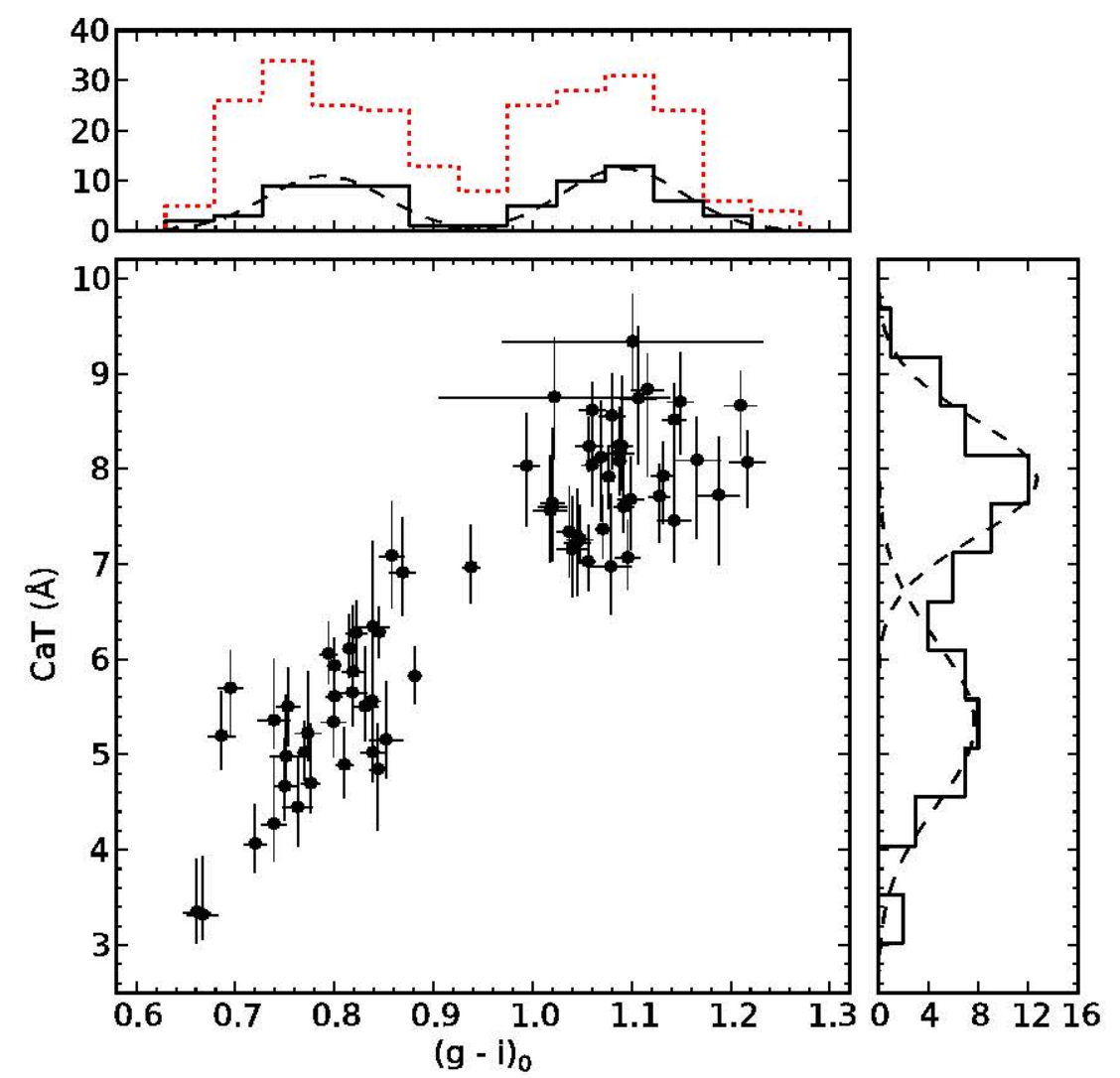

Figure 1. CaT index measurements vs. $(g-i)_{0}$. The solid and dotted histograms are the spectroscopic (71 GCs) and the entire, background-decontaminated, photometric ( 253 candidates) samples, respectively. The overall color distribution is well represented by the spectroscopic sample. Overplotted on the histograms are the best-fit Gaussians from a standard mixture-modeling analysis. Bimodality is preferred over unimodality at the $>99.9 \%$ and $99.8 \%$ levels for the color and CaT index distributions, respectively.

(A color version of this figure is available in the online journal.)

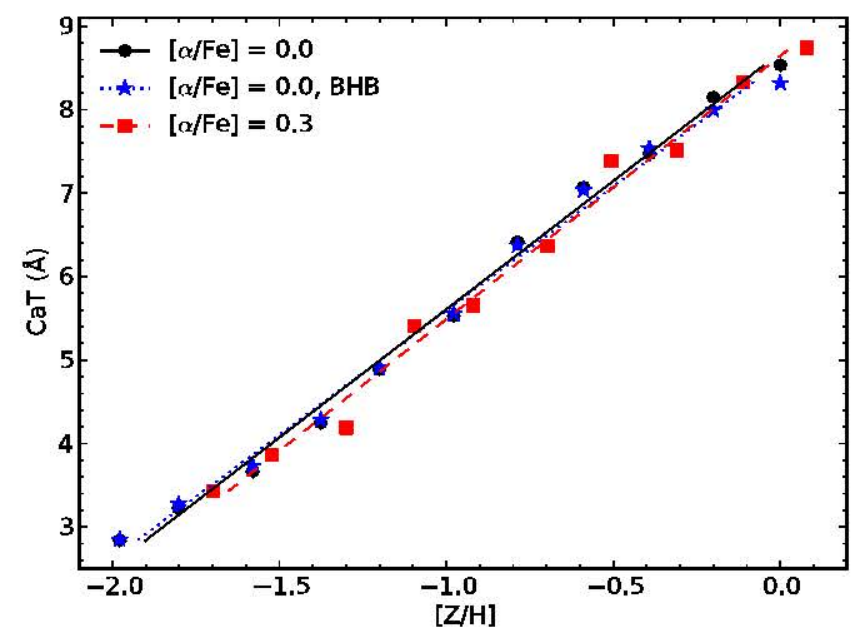

Figure 2. CaT index vs. metallicity based on the $13 \mathrm{Gyr}$ SSP models of Conroy et al. (2009) and Conroy \& Gunn (2010). Dotted line/star symbols are a model with an extreme blue HB at all metallicities and no $\alpha$-enhancement. Solid line/filled circles represent a purely red HB population, again with solar $[\alpha / \mathrm{Fe}]$. The dashed line/square symbols have $[\alpha / \mathrm{Fe}]=0.3$. Although blue HB stars do influence the Paschen lines, their effect on the CaT index is negligible. Our $\mathrm{CaT}$ measurements are insensitive to $[\alpha / \mathrm{Fe}]$.

(A color version of this figure is available in the online journal.)

appears to provide an acceptable (although not optimal) fit to the $[\mathrm{Z} / \mathrm{H}]$ data in Figure 3, it produces an MDF that is single-peaked with an extended metal-poor tail. This differs dramatically from the bimodal MDFs derived spectroscopically and from the Usher et al. (2012) relation.

\section{DISCUSSION}

Here we assess the cumulative evidence for and against GC metallicity bimodality and briefly discuss the relevance of GC metallicity subpopulations in unraveling the assembly histories of galaxies.

\subsection{Optical Spectroscopy}

Apart from the obvious example of our own Milky Way, where metallicity bimodality is irrefutable, there are several galaxies for which a reasonably large sample of GC metallicities have been measured directly from their spectra. M49 (NGC 4472), the brightest galaxy in the Virgo Cluster, was studied by Strader et al. (2007) who used the Keck/LRIS spectroscopy of Cohen et al. (2003) to estimate metallicities from Lick indices for 47 of its GCs. The resulting MDF was shown to be bimodal, and the locations of the red and blue peaks were shown to be consistent with expectations based on the GC metallicity-host galaxy mass relation (Strader et al. 2006; Peng et al. 2006).

More recently, Alves-Brito et al. (2011) used 112 high-quality Keck/DEIMOS spectra of confirmed GCs in the Sombrero galaxy (NGC 4594) to measure metallicities via the Brodie \& Huchra (1990) method. Again, the MDF is clearly bimodal with a KMM (mixture modeling algorithm; Ashman et al. 1994) probability $>90 \%$. Moreover, they find evidence for a linear transformation between metallicity and $(B-R)$ color.

The main weakness of the NGC 4472 work is the small sample size, especially as a fraction of the total GC system, and that only the bright end of the luminosity function is sampled. While the 


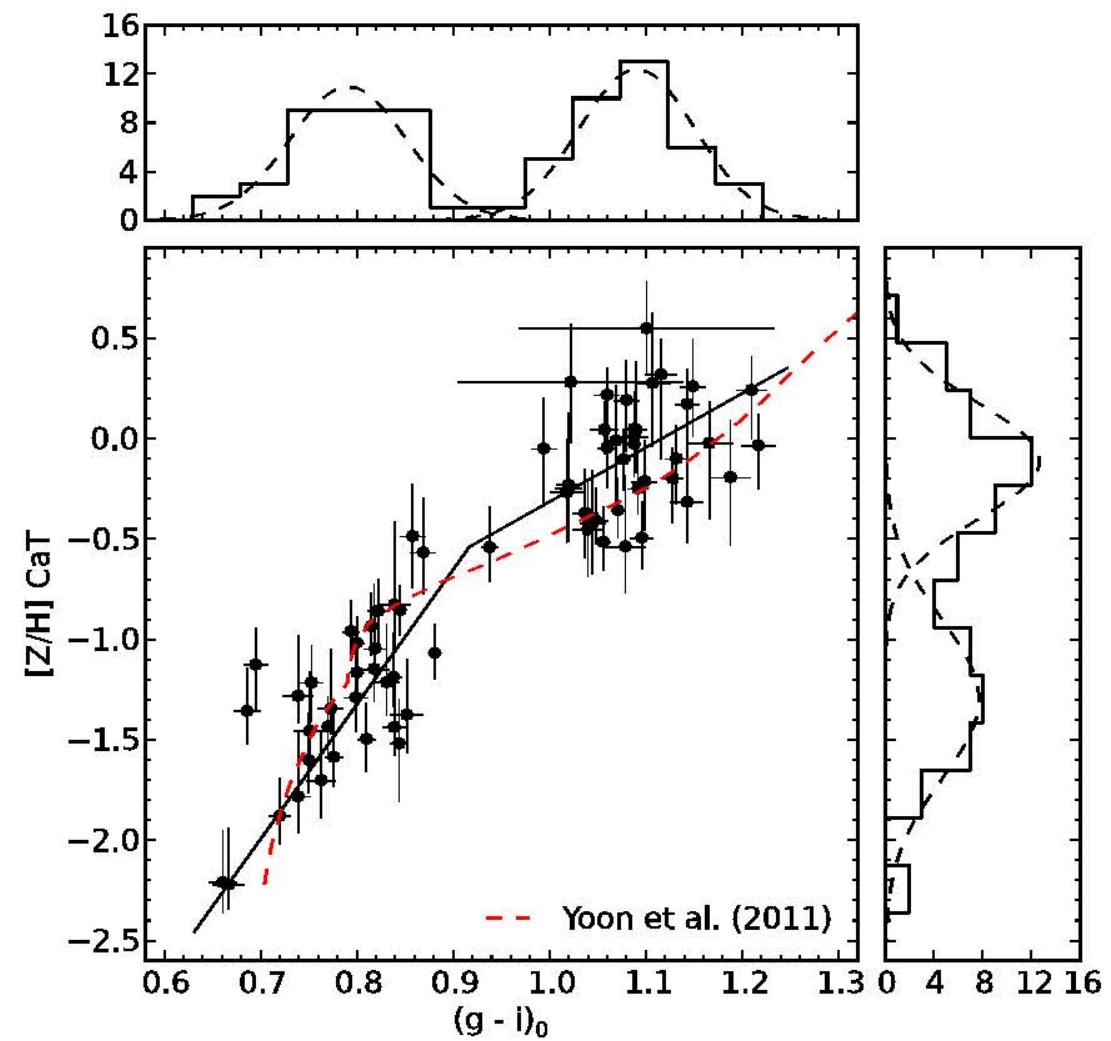

Figure 3. GC color-metallicity relation. Metallicities are derived from our CaT measurement for $71 \mathrm{GCs}$, using the CaT- $[\mathrm{Z} / \mathrm{H}]$ relation of Usher et al. (2012). The dashed line is the color-metallicity model relation of Yoon et al. (2011a) and the solid line is a two-component, best-fit linear color-metallicity relation that provides a better representation of the data.

(A color version of this figure is available in the online journal.)

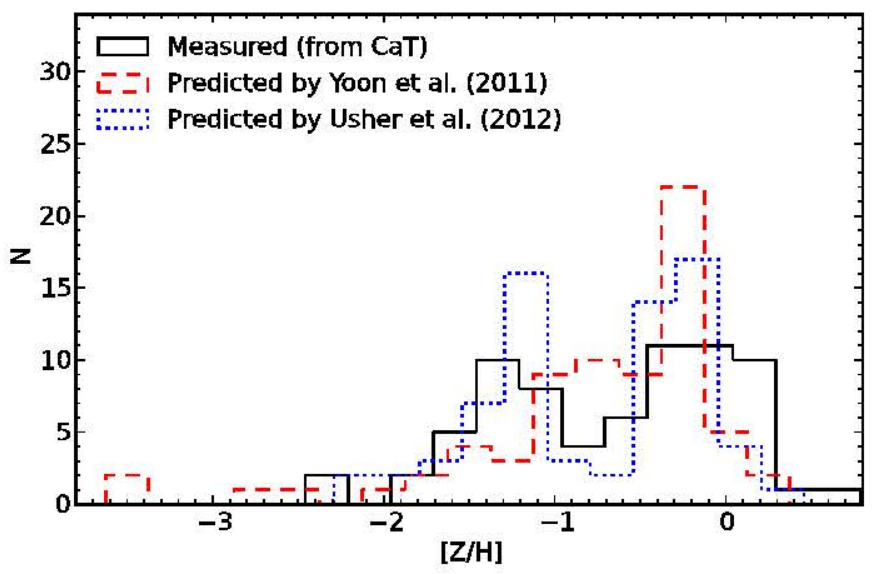

Figure 4. GC metallicity distribution functions. The solid line is the MDF derived from CaT measurements. It is bimodal at the $99.8 \%$ confidence level. The dashed and dotted lines are obtained from $(g-i)_{0}$ colors using the Yoon et al. (2011a) model color-[Z/H] relation and the Usher et al. (2012) empirical color $-[Z / H]$ relation, respectively.

(A color version of this figure is available in the online journal.)

sample in NGC 4594 is larger, the spectra are generally of lesser signal to noise than those of the 71 selected objects in NGC 3115. More critically, the NGC 3115 system is the definitive litmus test of metallicity bimodality because its color bimodality is much clearer than in either NGC 4472 or NGC 4594.

NGC 5128 (Cen A) is another galaxy with a well-studied GC system. Woodley et al. (2010) found bi/multimodality in the spectroscopically derived metallicities and colors of the old
GCs in their sample. However, NGC 5128 is known to be the remnant of a recent merger and has a population of relatively young GCs, presumed to have formed in that merger (Beasley et al. 2008). Although this galaxy is GC-rich and nearby, its complexity and special, post-merger status make it far from ideal as a bimodality benchmark.

Two well-studied GC-populous galaxies, M31 (Caldwell et al. 2011) and M87 (Cohen et al. 1998), have spectroscopic metallicity distributions that are not clearly bimodal, and for these the issue is not with sample size or signal to noise. Neither of these galaxies would fit our criterion for clear color bimodality. The peaks of the color distributions are poorly separated and the kurtoses are positive (M31: $\mathrm{DD}=1.47, k=0.23$; M87: $\mathrm{DD}=1.30, k=0.19$ ). Therefore, the lack of metallicity bimodality is to be expected. Moreover, only GCs significantly brighter than the mean were included in the Cohen et al. study. Interestingly though, in M87, Strader et al. (2011) found evidence from subsample kinematics and colors that multiple, old populations may be present, perhaps as a result of ongoing accretion of infalling satellite galaxies hosting GC systems with a range of masses and hence colors and metallicities. In general, the extended formation histories of central cluster galaxies like M87 may be expected to produce more complex GC systems, especially when studied in detail. M31 is also actively accreting satellite galaxies and their associated GCs (Mackey et al. 2010), although it is not clear whether this recent accretion history is typical of or unusual for massive disk galaxies.

An important point in the context of this work is that spectroscopically inferred MDFs, whether unimodal or multimodal, do 
not appear to be well reproduced by application of the Yoon et al. models. For example, in M31, using the photometry of Peacock et al. (2010) and the spectroscopic metallicities of Caldwell et al. (2011), we find that the K-S test gives a probability of $4 \times 10^{-8}$ that the metallicities predicted by the Yoon et al. models are drawn from the same distribution as the spectroscopic metallicities. A generic result from the Yoon et al. models is that they produce a single skewed metallicity distribution with a broad peak, steeply declining at the metal-rich end and with a long tail to metal-poor clusters. Usher et al. (2012) show that the observed $[\mathrm{Z} / \mathrm{H}] \mathrm{GC}$ distributions for 11 early-type galaxies are not as skewed or peaky as the Yoon et al. models would predict.

\subsection{Near-infrared Colors}

Near-infrared colors, primarily reflecting the temperatures of stars on the red giant branch, should be less sensitive to the distribution of stars on the HB. Thus, a unimodal metallicity distribution should map into a unimodal near-infrared color distribution, and the detection of bimodality in a near-infrared color distribution would strongly imply an underlying bimodal metallicity distribution. Chies-Santos et al. (2012) argued that $K$-band photometry is key to a clean discrimination of metallicity, but their detailed modeling of photometric scatter showed how difficult it is to obtain the sample size and data quality needed to reveal the effect. The difficulty is underscored in M87, where Kundu \& Zepf (2007) found clear GC color bimodality in $I-H$, based on NICMOS observations, while Chies-Santos et al. (2012) failed to find the effect in $g-K$ with a larger sample.

Although NGC 1399 shows significant GC bimodality in optical colors, Blakeslee et al. (2012) found it to be unimodal in $I-H$. A worry is that their inferred MDF does not seem to be consistent with results from the actual spectroscopic MDFs of other galaxies, as these consistently have much more significant metal-poor subpopulations than the MDFs produced from photometry using nonlinear color-metallicity relations. Spectroscopically derived metallicities are urgently needed for NGC 1399 GC system. Chies-Santos et al. found that the $g-K$ color distribution of NGC 4649 (M60) is best described by a bimodal distribution (KMM probability $>95 \%$ ), consistent with metallicity bimodality in this galaxy's GC system. Using Spitzer observations, Spitler et al. (2008) also found bimodal optical/mid-IR $(3.6 \mu \mathrm{m})$ color distributions in the GC systems of NGC 5128 and NGC 4594.

\subsection{Multiple GC Subpopulations and Galaxy Formation}

The well-established correlations between GC color and host galaxy properties (Forbes et al. 1997; Larsen et al. 2001; Strader et al. 2006; Peng et al. 2006) must be linked directly to metallicity effects to establish GCs as chemodynamical tracers of the star formation and assembly processes that shaped galaxies over cosmic time.

Several lines of evidence, including spatial and metallicity distributions and specific frequencies, have led to suggestions that metal-poor blue GCs are formed in dark matter halos at the earliest times and are associated with metal-poor galaxy halos (Strader et al. 2005; Forbes et al. 2012), while the metal-rich red GCs closely trace the buildup of galaxy bulges (Forbes et al. 2001; Strader et al. 2005; Pota et al. 2012).

The observed bimodality might arise naturally in the hierarchical merging paradigm as a consequence of the fundamental relation between GC metallicity and host galaxy mass (Muratov \& Gnedin 2010), or it may be the result of a temporary truncation of GC formation at high redshift (Brodie \& Strader 2006).

Regardless of the eventual viability of these scenarios, the linkage of GC and galaxy properties can be expected to provide fundamental, unique constraints on galaxy formation in a hierarchical merging framework.

\section{SUMMARY AND CONCLUSIONS}

$\mathrm{CaT}$ indices and $g i$ colors have been measured for $71 \mathrm{GCs}$ with high signal-to-noise spectra in NGC 3115, a nearby S0 galaxy with a populous GC system that exhibits the most strongly bimodal color distribution of any known GC system. The CaT index has been shown to be effectively insensitive to $\mathrm{HB}$ morphology and $\alpha$-enhancement over a range of metallicities from $[\mathrm{Z} / \mathrm{H}]=-2.0$ to $[\mathrm{Z} / \mathrm{H}]=0.0$ using new stellar population models. CaT correlates closely with $[Z / H]$ over the range of interest for GCs; it is an excellent proxy for metallicity in such systems. Color and CaT (and hence metallicity) are shown to be bimodal for NGC 3115 GCS with high statistical significance ( $>99.9 \%$ and $99.8 \%$, respectively).

The suggestion of Yoon et al. (2006) that the underlying metallicity distributions of GC systems are unimodal, and that the observed color bimodality is an artifact of strongly nonlinear color-metallicity transformations, is not confirmed in this galaxy. While this result does not necessarily apply to all early-type galaxies, it does lend considerable weight to the mounting evidence from direct MDFs that multiple GC metallicity subpopulations are common. The fact that a variety of optical colors and the easily measured $\mathrm{CaT}$ index are excellent indicators of metallicity provides us with a key diagnostic for unraveling the star formation and assembly histories of galaxies.

Data presented herein were obtained at the W. M. Keck Observatory, which is operated as a scientific partnership among Caltech, UC, and NASA. Based on data collected at Subaru (operated by NAOJ) via Gemini time exchange (GN-2008A-C-12). This work was supported by the NSF through grants AST-0808099, AST-0909237, and AST-1109878.

\section{REFERENCES}

Alves-Brito, A., Hau, G. K. T., Forbes, D. A., et al. 2011, MNRAS, 417, 1823 Armandroff, T. E., \& Zinn, R. 1988, AJ, 96, 92

Arnold, J. A., Romanowsky, A. J., Brodie, J. P., et al. 2011, ApJ, 736, L26 Ashman, K. M., Bird, C. M., \& Zepf, S. E. 1994, AJ, 108, 2348

Beasley, M. A., Bridges, T., Peng, E., et al. 2008, MNRAS, 386, 1443 Blakeslee, J. P., Cho, H., Peng, E. W., et al. 2012, ApJ, 746, 88

Brodie, J. P., \& Huchra, J. P. 1990, ApJ, 362, 503

Brodie, J. P., \& Strader, J. 2006, ARA\&A, 44, 193

Caldwell, N., Schiavon, R., Morrison, H., Rose, J. A., \& Harding, P. 2011, AJ, 141,61

Cenarro, A. J., Gorgas, J., Cardiel, N., Vazdekis, A., \& Peletier, R. F. 2002, MNRAS, 329, 863

Chies-Santos, A. L., Larsen, S. S., Cantiello, M., et al. 2012, A\&A, 539, A54

Cohen, J. G., Blakeslee, J. P., \& Côté, P. 2003, ApJ, 592, 866

Cohen, J. G., Blakeslee, J. P., \& Ryzhov, A. 1998, ApJ, 496, 808

Conroy, C., \& Gunn, J. E. 2010, ApJ, 712, 833

Conroy, C., Gunn, J. E., \& White, M. 2009, ApJ, 699, 486

Faifer, F. R., Forte, J. C., Norris, M. A., et al. 2011, MNRAS, 416, 155

Forbes, D. A., Brodie, J. P., \& Grillmair, C. J. 1997, AJ, 113, 1652

Forbes, D. A., Brodie, J. P., \& Larsen, S. S. 2001, ApJ, 556, L83

Forbes, D. A., Ponman, T., \& O’Sullivan, E. 2012, MNRAS, 425, 66

Foster, C., Forbes, D. A., Proctor, R. N., et al. 2010, AJ, 139, 1566

Foster, C., Spitler, L. R., Romanowsky, A. J., et al. 2011, MNRAS, 415, 3393

Harris, W. E., Whitmore, B. C., Karakla, D., et al. 2006, ApJ, 636, 90

Kundu, A., \& Zepf, S. E. 2007, ApJ, 660, L109

Larsen, S. S., Brodie, J. P., Huchra, J. P., Forbes, D. A., \& Grillmair, C. J. 2001, AJ, 121, 2974 
Mackey, A. D., Huxor, A. P., Ferguson, A. M. N., et al. 2010, ApJ, 717, L11

Mieske, S., Jordán, A., Côté, P., et al. 2006, ApJ, 653, 193

Muratov, A. L., \& Gnedin, O. Y. 2010, ApJ, 718, 1266

Ostrov, P., Geisler, D., \& Forte, J. C. 1993, AJ, 105, 1762

Peacock, M. B., Maccarone, T. J., Knigge, C., et al. 2010, MNRAS, 402, 803

Peacock, M. B., Zepf, S. E., Maccarone, T. J., \& Kundu, A. 2011, ApJ, 737, 5

Peng, E. W., Côté, P., Jordán, A., et al. 2006, ApJ, 639, 838

Pota, V., Forbes, D. A., Brodie, J. P., et al. 2012, MNRAS, accepted (arXiv:1209.4351)

Puzia, T. H., Kissler-Patig, M., Thomas, D., et al. 2005, A\&A, 439, 997

Richtler, T. 2006, Bull. Astron. Soc. India, 34, 83

Searle, L., \& Zinn, R. 1978, ApJ, 225, 357
Spitler, L. R., Forbes, D. A., \& Beasley, M. A. 2008, MNRAS, 389, 1150

Strader, J., Beasley, M. A., \& Brodie, J. P. 2007, AJ, 133, 2015

Strader, J., Brodie, J. P., Cenarro, A. J., Beasley, M. A., \& Forbes, D. A. 2005, AJ, 130,1315

Strader, J., Brodie, J. P., Spitler, L., \& Beasley, M. A. 2006, AJ, 132, 2333

Strader, J., Romanowsky, A. J., Brodie, J. P., et al. 2011, ApJS, 197, 33

Tonry, J. L., Dressler, A., Blakeslee, J. P., et al. 2001, ApJ, 546, 681

Usher, C., Forbes, D. A., Brodie, J. P., et al. 2012, arXiv: 1207.6402

Woodley, K. A., Harris, W. E., Puzia, T. H., et al. 2010, ApJ, 708, 1335

Yoon, S.-J., Lee, S.-Y., Blakeslee, J. P., et al. 2011a, ApJ, 743, 150

Yoon, S.-J., Sohn, S. T., Lee, S.-Y., et al. 2011b, ApJ, 743, 149

Yoon, S.-J., Yi, S. K., \& Lee, Y.-W. 2006, Science, 311, 1129

Zepf, S. E., \& Ashman, K. M. 1993, MNRAS, 264, 611 